\title{
Information-Efficient Metagrating for Transverse-Position Metrology
}

\author{
Zheng Xi, ${ }^{*}$ Sander Konijnenberg, ${ }^{\dagger}$ and H.P. Urbach \\ Optics Research Group, Delft University of Technology Delft, Netherlands
}

(Received 23 April 2019; revised 18 February 2020; accepted 20 May 2020; published 9 July 2020)

\begin{abstract}
We introduce an optimal metagrating design for transverse-position metrology in presence of photon shot noise. The proposed working principle is closely related to the formation of a phase vortex in the diffraction orders in the parameter space. Using the topological robustness, we optimize the design and compress all the transverse-position information around a certain point into a small number of detected photons, saturating the shot-noise limit had all the photons used for probing the position been detected. The current scheme avoids the problem of detector saturation in the presence of high probe power while maintaining all the information detected, allowing one to make full use of the high power that is available. Besides, the direct link between the resonant property in the unit cell and the conditions to achieve the bound is given: one with the zeroth dipole resonance and the other one with the anapole condition of the first dipole. The connection between the metagrating design and the optimization using topological robustness along with the fundamental precision limit using classical light gives new insights in all of these fields.
\end{abstract}

DOI: 10.1103/PhysRevApplied.14.014026

\section{INTRODUCTION}

One particular important research area in optics is the development of sensitive metrology methods to measure a very small displacement around a certain point [1-14]. This problem is fundamental to pushing the limits of fields such as interferometry [7-10], metrology [6], and superresolution microscopy [1-4]. Measuring a small displacement amounts to a parameter-estimation problem in the presence of measurement noise, which can be due to either imperfections of the setup or, more fundamentally, the intrinsic fluctuations due to photon shot noise using coherent light, which scales as $1 / \sqrt{N_{\text {probe }}}$, with $N_{\text {probe }}$ being the number of photons used to probe the change in position per unit time. One might intuitively think that the best measurement scheme is to detect all $N_{\text {probe }}$ photons, since no photons and thus no information about the displacement are lost. But this scheme soon runs into a problem when one tries to push the precision of the measurement by adding more probe photons: while extremely high optical powers are available (e.g., from several hundred watts to a few kilowatts at the beams splitter inside an interferometer) $[7,15,16]$, most fast photon detectors do not allow the direct exposure of such high powers [17]. Furthermore, the detectors are more sensitive at relatively low incoming powers. If one attenuates the number of photons $N_{\text {probe }}$ interacting with the measurement device, one throws

\footnotetext{
*z.xi@tudelft.nl

${ }^{\dagger}$ On leave from Optics Research Group at Delft University of Technology, now at ASML Research.
}

away information about the displacement. Thus an important question to be answered is as follows: Can one recover all the information contained in a large number of probe photons $\left(N_{\text {probe }}\right)$ per unit time by detecting a small fixed number of detected photons $\left(N_{\text {det }}\right)$ per unit time set by the limit of the detector? In other words, how can one develop an information-efficient measurement that compresses the full measured information into a small number of detection events?

This question has been answered in the development of large-scale interferometers $[7,15,16]$, which are used to measure extremely small longitudinal displacement. If the operating point of the interferometer is set near the socalled dark fringe of the Michelson interferometer, almost all the information about the longitudinal displacement is compressed into a small number of detected photons $\left(N_{\text {det }}\right)$, allowing one to make full use of the very high power inside the interferometer to increase the measurement precision. Moreover, advanced power-recycling techniques allow one to recycle all the undetected light to increase the effective number of probe photons, leading to a significant improvement compared with the case that one detects all the photons directly [18-20]. However, as mentioned, this scheme is used mainly for probing longitudinal displacement along the beam propagation direction.

Recently, a promising approach combing knowledge from structured light and nanophotonic resonant structures was proposed for transverse-position metrology [1113,21,22]. A distinct feature of resonant nanostructures containing balanced multipole resonances is the effect of directional scattering, known as the Huygens source 
fulfilling Kerker's condition [23,24]. When combined with structured light illumination, this directional scattering condition is highly dependent on the local vectorial field distributions. If designed properly, a sharply switched scattering directionality can be achieved when the nanostructures are displaced slightly from the origin of interest. It was demonstrated recently that the far-field directionality can be used to extract the transverse location of nanostructures $[11-13,21,22]$. Because this scheme detects only parts of the totally scattered light, one would expect loss of information. Thus, an important question remains unanswered: How can one use this scheme to optimize the illumination and the resonances excited to achieve the shot-noise limit for transverse-position metrology in an information-efficient way?

In this work, we propose an optimal resonant metagrating consisting of meta-atoms with specifically engineered scattering properties [25-28] as an information-efficient tool for transverse-position measurement. The position information of the metagrating around a certain point inside an illumination field is encoded into the reflected diffraction orders. By writing the field scattered by the unit cell of the metagrating as a superposition of fields produced by a set of multipoles, we show that at the resonance of these multipoles, the reflected order of interest has a phase vortex as a function of certain design parameters. We further use the topological stability of the phase vortex to tune the design parameters to maximize the information contained in the detected photons, allowing one to achieve the shot-noise limit set by $N_{\text {probe }}$, with $N_{\text {det }}$ much smaller than $N_{\text {probe }}$. Through the physical insights gained, two special resonant conditions are found to be related to the fundamental precision limit. The connection between the formation of a phase vortex due to the multipole resonances of the metagrating and the fundamental precision limit for position metrology opens new possibilities for the use of metadevices in metrology.

\section{WORKING PRINCIPLE OF THE METAGRATING}

Without any loss of generality, silicon nanowire $(n=$ 3.5 ) of radius $R$ with electric and magnetic resonances is chosen as the constituting element for the metagrating [26,29-33]. The metagrating is designed such that it has four diffraction orders as shown in Fig. 1(a). It is placed inside an interference field produced by two plane waves with an initial phase shift of $\pi$ as shown in Fig. 1(b). The wavelength used is $650 \mathrm{~nm}$ throughout this paper. The illumination of the metagrating is chosen such that the $x$ component of the incident wave vectors satisfies $k_{x}=\pi / P$, where $P$ is the pitch of the metagrating. Under this condition, the two reflected orders $P_{+}$and $P_{-}$(each produced by a combination of the zeroth and first reflection orders) are parallel to the incident angles. The metagrating

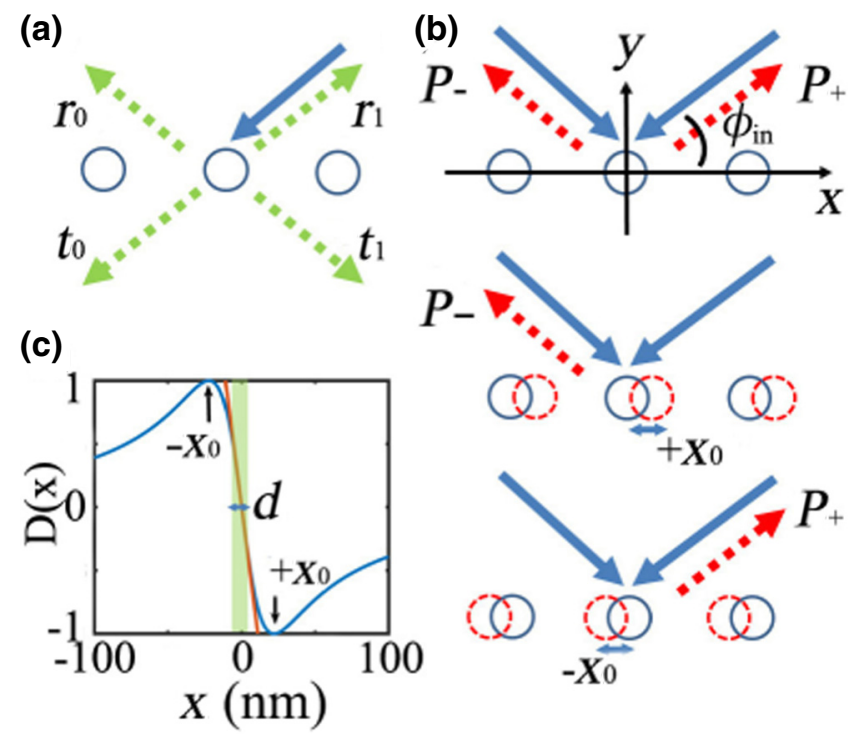

FIG. 1. (a) The metagrating design consisting of a periodic array of cylindrical nanowires. The incident fields are represented by the blue arrows with either TE or TM polarization. For each incident field, there are four diffraction orders, as indicated by the dashed green lines. (b) When the metagrating is placed inside an interference field formed by two plane waves of initial phase difference $\pi$, there are two reflected orders $P_{+}$ and $P_{-}$. Depending on the displacement indicated by the dashed red circles, $P_{+}$and $P_{-}$disappear at $+x_{0}$ and $-x_{0}$, respectively. (c) Normalized power difference as a function of displacement. The illumination is TM polarization with $\phi_{\text {in }}=60^{\circ}, R=70 \mathrm{~nm}$, and for this design $x_{0}=22 \mathrm{~nm}$. The green-shaded region $d$ is the linear region within which $D(x)$ can be fitted by the red line.

is designed to make $P_{+}$and $P_{-}$vanish when displaced by $+x_{0}$ and $-x_{0}$, respectively. Because the displacement $x_{0}$ is equivalent to adding a phase shift of $2 k_{x} x_{0}$ to one of the two beams, this requirement can be expressed as

$$
r_{0}-r_{1} e^{-2 i k_{x} x_{0}}=0
$$

where $r_{0}$ and $r_{1}$ are the reflection coefficients of the zeroth and first orders and the minus sign is due to the initial phase shift of $\pi$. Assuming a total input power of $\mathscr{P}_{0}$, the powers of the two diffraction orders at position $x$ are

$$
\begin{aligned}
& \mathscr{P}_{+}=2\left|r_{0}\right|^{2} \mathscr{P}_{0} \sin ^{2} k_{x}\left(x-x_{0}\right), \\
& \mathscr{P}_{-}=2\left|r_{0}\right|^{2} \mathscr{P}_{0} \sin ^{2} k_{x}\left(x+x_{0}\right) .
\end{aligned}
$$

In Fig. 1(c) we show the normalized power difference of the two reflected orders defined by $D(x)=\left(\mathscr{P}_{+}-\right.$ $\left.\mathscr{P}_{-}\right) /\left(\mathscr{P}_{+}+\mathscr{P}_{-}\right)$. The value of position $x$ can be inferred by measuring $D(x)$ without scanning, provided that $x$ is in the interval $\left[-x_{0}, x_{0}\right]$. For a region $d$ shown in Fig. 1(b), $D(x)$ can be linearized with the slope given by $D^{\prime}(0)=$ $-2 / x_{0}$. A smaller $x_{0}$ leads to a larger slope and thus a higher sensitivity. 


\section{FISHER INFORMATION AND CRAMÉR-RAO LOWER BOUND FROM THE DETECTED PHOTONS}

Even in the ideal case of no background noise, the quantum property of light introduces fluctuations in the detected signal, which sets a limit on the precision that can be achieved. This limit is often described using a lower bound on the measurement uncertainty called the quantum Cramér-Rao lower bound (CRLB), which is the inverse of the square root of quantum Fisher information. For classical light, the bound is generally expressed as the shot-noise limit and is optimized over all the measurement schemes and estimators for use of the coherent state.

For our case, because a displacement $x$ from the origin is equivalent to adding a phase shift of $e^{2 i k_{x} x}$ to one of the incoming beams, the shot-noise limit for position estimation can be easily derived from the well-known shot-noise limit for phase estimation, which is

$$
\sigma_{x}^{\mathrm{SNL}}=\sigma_{\phi}^{\mathrm{SNL}} / 2 k_{x}=\frac{1}{2 k_{x} \sqrt{N_{\text {probe }}}},
$$

where $N_{\text {probe }}=\mathscr{P} / \hbar \omega$ is the total number of probe photons used per unit time to probe the position. This limit serves as a criterion to evaluate the performance of different measurement schemes, in our case different metagrating designs. The optimal metagrating design should attain this limit by detecting $N_{\text {det }}$ photons, with $N_{\text {det }} \ll N_{\text {probe }}$.

However, as noted, the shot-noise limit is optimized over all metagrating designs and is thus independent of the detailed design parameters of the metagrating. To gain physical insights into the links between the detailed properties of the metagrating and the shot-noise limit, we next consider the Fisher information contained in the detected photons and derive the Cramér-Rao bound from these detected photons, which is dependent on the properties of the metagrating. By comparison of the Cramér-Rao bound in the detected photons and the shot-noise limit, knowledge of the design requirements for the metagrating can be gained.

We consider the Fisher information contained in the reflected photons because we detect only them. We first release the condition that Eq. (1) imposes and consider a generalized grating with reflection coefficients $\left|r_{0}\right|$ and $\left|r_{1}\right| e^{-i \Delta_{01}}$ with a phase difference $\Delta_{01}$. In this case, the powers contained in the two reflected orders are

$$
\begin{aligned}
& \mathscr{P}_{+}(x)=A_{0} \mathscr{P}\left[1-V \cos \left(2 k_{x} x-\Delta_{01}\right)\right], \\
& \mathscr{P}_{-}(x)=A_{0} \mathscr{P}\left[1-V \cos \left(2 k_{x} x+\Delta_{01}\right)\right],
\end{aligned}
$$

where $A_{0}=\left(r_{0}^{2}+r_{1}^{2}\right) / 2$ and $V=\left|2 r_{0} r_{1}\right| /\left(r_{0}^{2}+r_{1}^{2}\right)$ is the visibility.

Under photon shot noise, the number of detected photons per unit time follows a Poisson distribution with mean
$N_{\mathrm{det}}=\left[\mathscr{P}_{+}(x)+\mathscr{P}_{-}(x)\right] / \hbar \omega$. One can use Fisher information to characterize the bound in the uncertainty of the retrieved position. By applying the definition of Fisher information and the chain rule [34], one arrives at

$$
I_{\mathrm{det}}(x)=\frac{1}{N_{\mathrm{det}}}\left(\frac{d N_{\mathrm{det}}}{d x}\right)^{2} .
$$

If one is interested in knowing the position $x=0$ or measuring very small displacements around this point,

$$
\begin{aligned}
\lim _{x \rightarrow 0} I_{\text {det }}(x) & =\lim _{x \rightarrow 0} 8 A_{0} k_{x}^{2} N_{\text {probe }} \frac{V^{2} \sin ^{2}\left(2 k_{x} x\right) \cos ^{2} \Delta_{01}}{1-V \cos \left(2 k_{x} x\right) \cos \Delta_{01}} \\
& \leq 16\left|r_{0}\right|^{2} k_{x}^{2} N_{\text {probe }},
\end{aligned}
$$

which reaches its maximum when $V=1$ and $\Delta_{01}=0$. The uncertainty bound $\sigma^{\mathrm{CRLB}}(0)$ for position estimation around this point is thus

$$
\begin{aligned}
\sigma^{\mathrm{CRLB}}(0) & =\frac{1}{\sqrt{I_{\mathrm{det}}(0)}} \\
& \geq \frac{1}{4\left|r_{0}\right| k_{x} \sqrt{N_{\text {probe }}}},
\end{aligned}
$$

which is dependent on the reflection coefficient of the grating used. Comparing this with the shot-noise limit in Eq. (3), which is the bound irrespective of the specific grating parameters, we have an additional requirement for the metagrating design such that $\left|r_{0}\right|=1 / 2$.

Through this detailed analysis of the CRLB for different metagrating designs and its comparison with the shot-noise limit, which is the optimal limit that can be achieved for any grating design and any unbiased estimators, we summarize the two requirements for the metagrating design:

(a) $V=1$ and $\Delta_{01}=0$. This condition is equivalent to the requirement of Eq. (1) with $x_{0} \ll k_{x}$ by noting $2 k_{x} x_{0}=$ $\Delta_{01}$.

(b) The reflection coefficient should be $\left|r_{0}\right|=1 / 2$.

Once these two conditions are fulfilled, we can say the metagrating is optimal for measurement of very small displacement under photon shot noise in a sense that it attains the shot-noise limit given by Eq. (3). Moreover, according to Eq. (2), as $x_{0}$ becomes smaller, the detected power around $x=0$ in $\mathscr{P}_{+}$and $\mathscr{P}_{-}$is much less than the probe power $\mathscr{P}$. In the case of very large $\mathscr{P}$, the scheme avoids the problem of detector saturation. Thus, it is information efficient.

\section{DESIGN OF THE METAGRATING}

The question now is how to design a metagrating that fulfills the above requirements. We use multipole scattering theory to describe our system as it relates directly 
the diffraction properties to the different multipole components, which provides valuable insights into the optimized design.

\section{A. Multipole scattering theory}

The total field in the far field of the grating can be written as

$$
U=e^{i k_{\mathrm{inx}} x+i k_{\mathrm{in} y} y}+\sum_{\mu} C_{\mu}\left(\phi_{\mu}\right) G\left(\phi_{\mu}, \phi_{\mathrm{in}}\right) e^{i k_{\mu x} x+i k_{\mu y} y},
$$

where $C_{\mu}\left(\phi_{\mu}\right)=2 / k_{0} P \sin \phi_{\mu}$, with $P$ being the pitch of the grating. The first term corresponds to the incident field with incident wavevectors $\vec{k}_{\text {in }}=\left(k_{\text {inx } x}, k_{\text {iny } y}\right)$ and the second term corresponds to the scattered field from the grating. $\phi_{\mu}$ is the diffraction angle of order $\mu$, and $\phi_{\text {in }}$ is the incident angle. $G\left(\phi_{\mu}, \phi_{\text {in }}\right)$ is the multiple-scattering Green's function along the $\phi_{\mu}$ direction and can be expressed using multipole decomposition:

$$
G\left(\phi_{\mu}, \phi_{\mathrm{in}}\right)=\sum_{m=-\infty}^{+\infty} A_{m}\left(\phi_{\mathrm{in}}\right) e^{i m \phi_{\mu}}
$$

with $A_{m}$ corresponding to the multiple-scattered multipole coefficients. They can be constructed from the isolated scattering amplitude $a_{m}$ for a single nanowire:

$$
A_{m}\left(\phi_{\text {in }}\right)=a_{m}\left(e^{i m \phi_{\text {in }}}+\sum_{n=-\infty}^{+\infty} A_{n}\left(\phi_{\text {in }}\right) \mathscr{L}(n, m)\right),
$$

where $\mathscr{L}(n, m)$ is the lattice sum, which takes full account of the multiple scattering between nanowires at different unit cells. From these, one can write down the expressions for the reflection and transmission coefficients of diffraction order $\mu$ :

$$
\begin{aligned}
& r_{\mu}=C_{\mu}\left(\phi_{\mu}\right) G\left(\phi_{\mu}, \phi_{\text {in }}\right), \\
& t_{\mu}=\delta_{\mu 0}+C_{\mu}\left(2 \pi-\phi_{\mu}\right) G\left(2 \pi-\phi_{\mu}, \phi_{\text {in }}\right) .
\end{aligned}
$$

It can be seen that the reflection coefficient of a certain order is fully determined by the Green's function along that direction. We substitute the reflection coefficients into Eq. (1):

$$
G\left(\phi_{0}, \phi_{\text {in }}\right)-G\left(\phi_{1}, \phi_{\text {in }}\right) e^{-2 i k_{x} x_{0}}=0,
$$

where $\phi_{0}=\pi-\phi_{\text {in }}$ and $\phi_{1}=\phi_{\text {in }}$ are the angles of the two reflected orders. For sufficiently small radii, one can keep only the first three terms $A_{-1}, A_{0}$, and $A_{1}$ in the expansion of $G$ :

$$
\begin{aligned}
& A_{0} \sin \left(\frac{\Delta_{01}}{2}\right)-i A_{1} \cos \left(\phi_{\text {in }}-\frac{\Delta_{01}}{2}\right) \\
& -i A_{-1} \cos \left(\phi_{\text {in }}+\frac{\Delta_{01}}{2}\right)=0,
\end{aligned}
$$

where $\Delta_{01}=2 k_{x} x_{0}$.

The above derivation works for both TE and TM polarization. For TM polarization, the term $A_{0}$ corresponds to the multiple-scattered linearly polarized magnetic dipole in the array. Because of the broken symmetry, the $A_{-1}$ and $A_{1}$ terms are different when $\phi_{\text {in }} \neq 90^{\circ}$. Since their scattered fields carry different topological charges -1 and +1 as shown in the insets in Fig. 2(a), they can be associated with the multiple-scattered left-rotating and right-rotating electric dipoles. For TE polarization, $A_{0}$ corresponds to the (a)

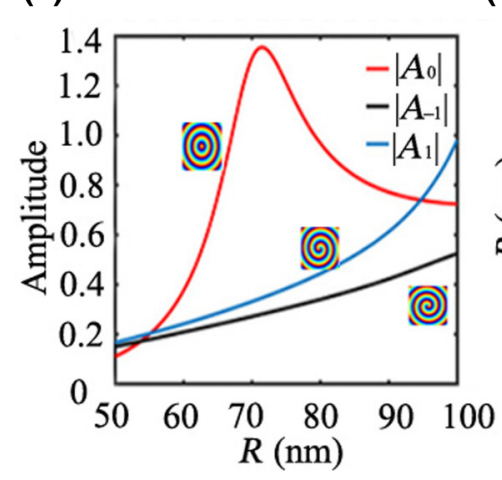

(b)

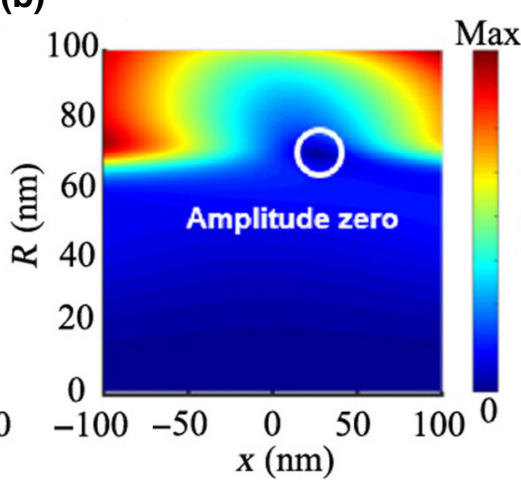

(c)

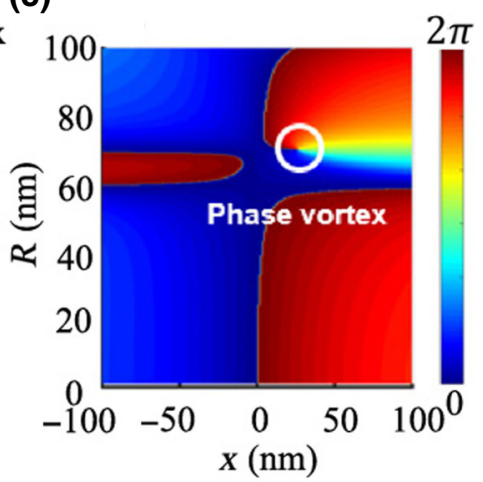

FIG. 2. (a) Amplitude of the first three multipole coefficients for $\phi_{\text {in }}=60^{\circ}$. The insets show the phase of the scattered field for each multipole for TM polarization. The reflection coefficient $\left|r_{0}\right|$ is also shown. A clear resonance can be seen around $R=70 \mathrm{~nm}$, around which $\left|r_{0}\right| \approx 0.5$. (b),(c) Absolute value and phase of the complex reflection amplitude of $P_{+}$when the metagrating of different radius $R$ is placed at different locations inside an interference field. A clear amplitude zero and a phase vortex can be seen at the location of the design fulfilling Eq. (1) with $x_{0}=22 \mathrm{~nm}$. The results are obtained with use of multipole scattering theory developed in Refs. $[35,36]$ and are double-checked by COMSOL MULTIPHYSICS simulation. 
multiple-scattered linearly polarized electric dipole, while $A_{-1}$ and $A_{1}$ correspond to the rotating magnetic dipoles.

On the left-hand-side of Eq. (12) is the complex amplitude of reflection order $P_{+}$at position $x=x_{0}$. To make this order vanish at $x_{0}$, there should be a proper balance of the different multipole components as indicated by Eq. (13). The term $i$ in front of $A_{1}$ and $A_{-1}$ indicates a phase difference of $\pi / 2$ is required. When one of the multipoles experiences a resonance, the phase around this resonance changes rapidly, by which this requirement can be fulfilled.

\section{B. Topological vortex in the design space}

To verify this, we show in Fig. 2(a) the behavior of different multipoles as a function of the radius $R$ of the nanowire for $\phi_{\text {in }}=60^{\circ}$, with the insets showing the phase map of each multipole component. The incident angle $\phi_{\text {in }}$ is chosen to be $60^{\circ}$ as in Fig. 1(c). A clear resonance in $A_{0}$ is seen at $R=70 \mathrm{~nm}$, which corresponds to the multiplescattered magnetic dipole resonance. In Figs. 2(b) and 2(c) the absolute value and phase of the complex amplitude of the reflection order $P_{+}$are shown as a function of position $x$ and radius $R$. At $x=22 \mathrm{~nm}$ and $R=70 \mathrm{~nm}$, the amplitude is zero, meaning that for these values Eq. (1) is satisfied, which is in agreement with the zero of $D(x)$ in Fig. 1(c). It is particularly interesting to look at the phase distribution around this zero-amplitude point. Because of the presence of the multiple-scattered magnetic dipole resonance in $A_{0}$, the phase changes very rapidly, forming a phase vortex of topological charge +1 in Fig. 2(c). It is at this phase-vortex point that the metagrating satisfies the design requirement. Because such a phase vortex is topologically stable [37-40], it follows that by continuous variation of parameters such as the incident angle $\phi_{\text {in }}$, Eq. (1) is again satisfied for some other $R$ and $x_{0}$ values. Therefore, we can use this topological robustness to minimize $x_{0}$. Besides, different incident polarizations have different multipole coefficients, which also influence how small $x_{0}$ can be obtained.

\section{Use of topological robustness to minimize $x_{0}$}

The results of exploring the above possibilities are summarized in Fig. 3(a). On the horizontal axes, the radius $R$ and incident angle $\phi_{\text {in }}$ are varied and the absolute value of the normalized power difference $D\left(x_{0}\right)$ is plotted as different colors at different $x_{0}$ values. Each red point in Fig. 3(a) corresponds to a good metagrating design with $\left|D\left(x_{0}\right)\right| \approx$ 1 . By continuous variation of $\phi_{\text {in }}$, the good design that corresponds to the formation of the phase vortex is always satisfied for some other $R$ and $x_{0}$ values.

There are three distinct curves giving good metagrating designs, which correspond to the trajectories of phase vortexes. The continuity of the curve confirms the topological robustness in the presence of a small perturbation. The origins of the three curves can be identified by comparing them with the position of multipole resonances as indicated in the plot. It can be seen that good designs can also be obtained from resonances with $m=-1,1$ for TE polarization. In this case, it is the resonances from the two rotating dipoles that provide the required $\pi / 2$ phase shift. A small change of $R$ gives a large change in $x_{0}$ for the $m=-1,1$ branch as follows from the dotted line in Fig. 3(a) (which is not continuous due to insufficient sampling in $R$ ). We therefore focus on the two $m=0$ branches.

Although an explicit expression for the dependence of $x_{0}$ on $R$ and $\phi_{\text {in }}$ is complicated, to make $x_{0}$ small, a smaller $R$ and a relatively large $\phi_{\text {in }}$ are needed. We restrict $\phi_{\text {in }}$ to be smaller than $70^{\circ}$ to represent the occurrence of a second reflection order. By imposing this constraint, the minimum $x_{0}$ is determined for each polarization. The normalized power difference $D(x)$ is plotted in Fig. 3(b) for both polarizations for the design of the minimum $x_{0}$. With TE polarization, a much smaller $x_{0}(0.08 \mathrm{~nm})$ can be achieved than with TM polarization $(15 \mathrm{~nm})$ meaning that a much higher probe power can be used for TE polarization than for TM polarization. It is further confirmed that for both cases a phase vortex occurs around the desired design shown in the insets in Fig. 3(b). (a)

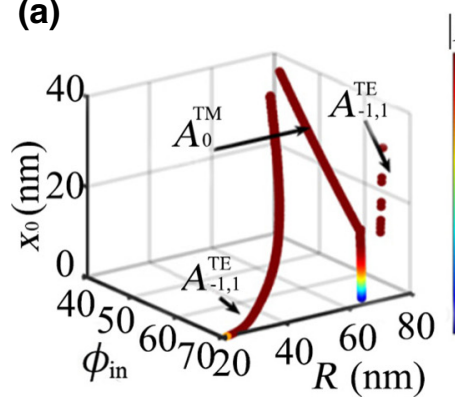

(b)

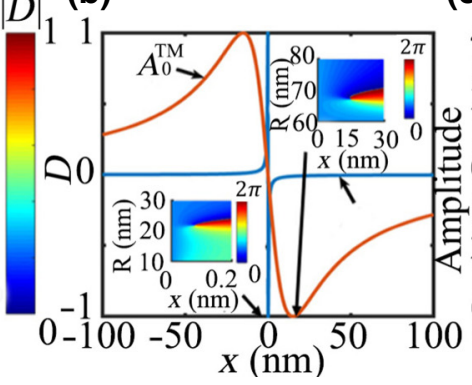

(c)

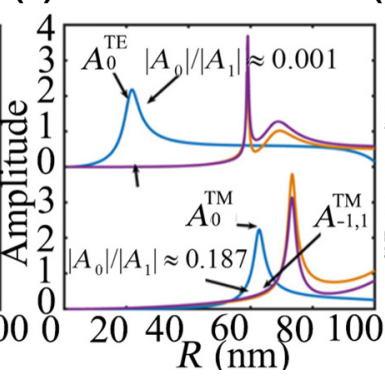

(d)

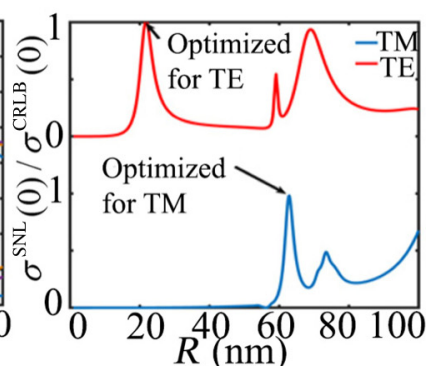

FIG. 3. (a) Achievable $x_{0}$ for different combination of $R$ and $\phi_{\text {in }}$ for both TE polarization and TM polarization using metagrating designs. (b) Absolute value of the normalized power difference $D(x)$ for TE and TM polarizations for the optimum design with minimum $x_{0}$. The insets show the formation of a phase vortex around the optimum design. (c) Multipole amplitude strength for TE and TM polarizations at minimum $x_{0}$. (d) Achievable $\sigma^{\mathrm{CRLB}}(0)$ for TE and TM polarizations. Designs with minimum $x_{0}$ saturate the shot-noise limit. 


\section{Fulfilling the requirement for reflection coefficient $\left|r_{0}\right|=1 / 2$}

It remains to be checked if the optimized design given above also fulfills the second requirement: that is, the reflection coefficient $\left|r_{0}\right|=1 / 2$.

To see this is indeed the case, we refer to Eq. (11). We note that the optimized design obtained above works at $A_{0}$ resonance. We assume at this resonance other higherorder $A_{m}$ terms are much smaller. In this case, we can approximate Eq. (11) using the contribution only from $A_{0}$ :

$$
\begin{aligned}
r_{0} & \approx C_{0}\left(\phi_{0}\right) A_{0}\left(\phi_{\text {in }}\right), \\
r_{1} & \approx C_{1}\left(\phi_{1}\right) A_{0}\left(\phi_{\text {in }}\right)=r_{0}, \\
t_{0} & \approx 1+C_{0}\left(2 \pi-\phi_{0}\right) A_{0}\left(\phi_{\text {in }}\right)=1-r_{0}, \\
t_{1} & \approx C_{1}\left(2 \pi-\phi_{1}\right) A_{0}\left(\phi_{\text {in }}\right)=-r_{0} .
\end{aligned}
$$

Furthermore, energy conservation requires that

$$
\left|r_{0}\right|^{2}+\left|r_{1}\right|^{2}+\left|t_{0}\right|^{2}+\left|t_{1}\right|^{2}=1
$$

which leads to $\left|r_{0}\right|=1 / 2$.

In Fig. 3(d), we plot the ratio of the shot-noise limit $\sigma^{\mathrm{SNL}}(0)$ given by Eq. (3) and the achievable uncertainty bound $\sigma^{\mathrm{CRLB}}(0)$ for the above designs under different polarizations at $\phi_{\text {in }}=70^{\circ}$. Because of the large $\left|A_{0} / A_{1}\right|$ ratio shown in Fig. 3(c), the shot-noise limit is achieved for TE polarization at $R=22 \mathrm{~nm}$ and approximately achieved for TM polarizaton at $R=62 \mathrm{~nm}$. The fact that the shotnoise limit is achieved proves the design is optimal.

\section{SATURATING THE SHOT-NOISE LIMIT NEAR THE ANAPOLE CONDITION}

Already from Eq. (13) one can gain some knowledge of the optimal design. We assume $x_{0} \ll k_{x}$ is fulfilled and $A_{1} \approx A_{-1}$, and then Eq. (13) can be simplified as

$$
k_{x} x_{0} \approx 2 i A_{1} / A_{0}
$$

There are two ways to make $x_{0}$ small. The first one is to work around the resonance of the zeroth dipole term $A_{0}$ as shown in the above analysis. The second one is to work around the point where $A_{1}$ becomes very small. We now discuss the second case.

Looking at Eq. (10), we note when the scattering coefficient $a_{1}$ becomes zero, the effect of the lattice sum becomes negligible. For the isolated nanowire, $a_{1}=0$ corresponds to the excitation of the anapole at which destructive interference of the dipole and the toroidal moments happen. At this condition $A_{-1}=A_{1}=0$, which makes the ratio $A_{1} / A_{0}$ zero. However, it does not satisfy the $\pi / 2$ phase requirement of Eq. (13). We therefore expect to see an optimal design slightly away from the anapole condition.

We redesign our unit cell using $\mathrm{Ag}$-core, $\mathrm{SiO}_{2}$-shell nanowire as shown in Fig. 4. The refractive indexes are

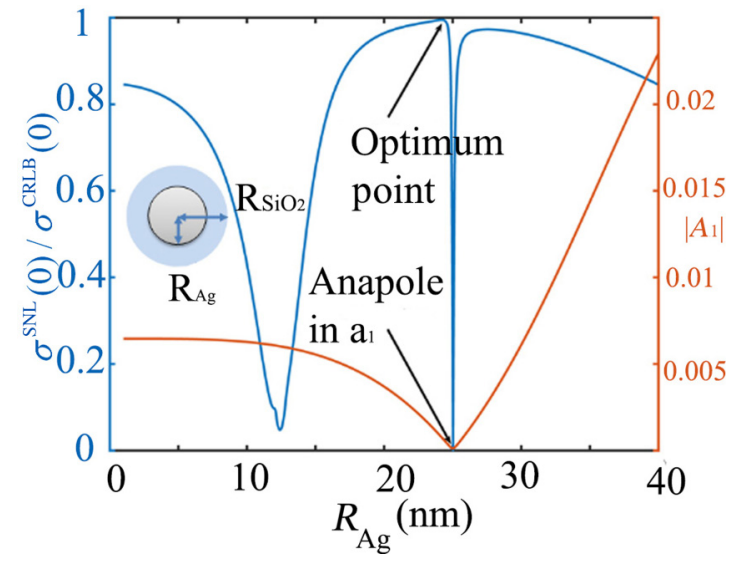

FIG. 4. Attaining the shot-noise limit near the anapole condition. The inset shows the structure of the unit-cell design.

chosen to be $n_{\mathrm{Ag}}=0.052225+4.4094 i$ and $n_{\mathrm{SiO}_{2}}=1.5$. We keep the incident angle $\phi_{\text {in }}=5^{\circ}$ and the outer radius $R_{\mathrm{SiO}_{2}}=50 \mathrm{~nm}$ fixed and vary the inner radius of the $\mathrm{Ag}$ core. We show in Fig. 4 the multipole coefficient $A_{1}$. An anapole excitation is clearly seen at $R_{\mathrm{Ag}}=25 \mathrm{~nm}$. We further plot the ratio of the shot-noise limit and the achievable uncertainty bound as a function of $R_{\mathrm{Ag}}$. It can be seen at the exact anapole condition that the uncertainty in retrieving $x=0$ is much larger than the shot-noise limit because the phase requirement is not fulfilled. It is also important to have the detected power slightly above the noise caused by other sources, thus minimizing the effect of imperfect measurement and maximizing the amount of information detected. If one moves away slightly from the anapole condition, the $A_{1}$ coefficient is still very small but the phase condition is fulfilled. The shot-noise limit is achieved as indicated by the optimal point in the plot.

\section{POWER RECYCLING}

Finally we discuss briefly the technique of power recycling and show how is it possible to combine it with our metagrating scheme to increase the measurement precision. This technique was firstly introduced by Drever [18] for the detection of gravitational waves. The key idea is basically to recycle all the noninformative photons that are not used for detection in the so-called bright port back into the interferometer and send them into a Fabry-Perot cavity enclosing the intereferometer which can be used for probing the phase shift again. In this way, the power inside the interferometer is boosted by the effective time that the photons are trapped inside the cavity, which is on the order of several hundred times due to typical loss of the cavity [16]; therefore, the number of effective probe photons is increased, allowing one to achieve an even-lower CRLB permitted by the shot-noise limit than in the case without power recycling. In our metagrating scheme, since we detect only the reflected photons, which are designed 
to have most of the information, the transmitted photons carry negligible information about the transverse position. Those photons in the transmitted orders can then be sent back to the metagrating again for power recycling, thereby increasing $N_{\text {probe }}$ to $g N_{\text {probe }}$, where $g$ is the gain factor introduced by the cavity enclosing the metagrating [18-20]. Thus, the sensitivity of the scheme is increased further. We emphasize this power-recycling scheme can be another major advantage of the current scheme besides efficient information compression.

\section{CONCLUSION}

In summary, we propose an optimal metagrating for high-precision transverse-position metrology under Poisson shot noise. By exploring the resonance and topological features of the metagrating, we show that the shot-noise limit can be achieved by detecting only a small number of photons used for probing the position, making the scheme information efficient. It is found that around the zeroth dipole resonance and the anapole condition of the first dipole, the shot-noise limit is achieved. We believe our metagrating design using topological robustness to achieve the shot-noise limit in an information-efficient way will result in new insights in both the field of nanophotonics and the field of nanometrology.

\section{ACKNOWLEDGMENT}

This work was funded through the EMPIR project 17FUN01-BeCOMe. The EMPIR initiative is cofunded by the European Union Horizon 2020 research and innovation program and the EMPIR participating states.

[1] Stefan W. Hell and Jan Wichmann, Breaking the diffraction resolution limit by stimulated emission: Stimulatedemission-depletion fluorescence microscopy, Opt. Lett. 19, 780 (1994).

[2] Michael J. Rust, Mark Bates, and Xiaowei Zhuang, Subdiffraction-limit imaging by stochastic optical reconstruction microscopy (storm), Nat. Methods 3, 793 (2006).

[3] Eric Betzig, George H. Patterson, Rachid Sougrat, O. Wolf Lindwasser, Scott Olenych, Juan S. Bonifacino, Michael W. Davidson, Jennifer Lippincott-Schwartz, and Harald F. Hess, Imaging intracellular fluorescent proteins at nanometer resolution, Science 313, 1642 (2006).

[4] Stefan W. Hell, Far-field optical nanoscopy, Science 316, 1153 (2007).

[5] Norman Bobroff, Recent advances in displacement measuring interferometry, Meas. Sci. Technol. 4, 907 (1993).

[6] Arie J. den Boef, Optical wafer metrology sensors for process-robust $\mathrm{cd}$ and overlay control in semiconductor device manufacturing, Surface Topography: Metrol. Prop. 4, 023001 (2016).

[7] Benjamin P. Abbott, Richard Abbott, T. D. Abbott, M. R. Abernathy, Fausto Acernese, Kendall Ackley, Carl Adams,
Thomas Adams, Paolo Addesso, and R. X. Adhikari, et al., Observation of Gravitational Waves from a Binary Black Hole Merger, Phys. Rev. Lett. 116, 061102 (2016).

[8] J. R. Gardner, M. L. Marable, G. R. Welch, and J. E. Thomas, Suboptical Wavelength Position Measurement of Moving Atoms Using Optical Fields, Phys. Rev. Lett. 70, 3404 (1993).

[9] David M. Giltner, Roger W. McGowan, and Siu Au Lee, Atom Interferometer Based on Bragg Scattering from Standing Light Waves, Phys. Rev. Lett. 75, 2638 (1995).

[10] M. Granata, C. Buy, R. Ward, and M. Barsuglia, HigherOrder Laguerre-Gauss Mode Generation and Interferometry for Gravitational Wave Detectors, Phys. Rev. Lett. 105, 231102 (2010).

[11] Martin Neugebauer, Paweł Woźniak, Ankan Bag, Gerd Leuchs, and Peter Banzer, Polarization-controlled directional scattering for nanoscopic position sensing, Nat. Commun. 7, 11286 (2016).

[12] Zheng Xi Lei Wei, Aurle Joseph Louis Adam, H. P. Urbach, and Luping Du, Accurate Feeding of Nanoantenna by Singular Optics for Nanoscale Translational and Rotational Displacement Sensing, Phys. Rev. Lett. 117, 113903 (2016).

[13] Ankan Bag, Martin Neugebauer, Paweł Woźniak, Gerd Leuchs, and Peter Banzer, Transverse Kerker Scattering for Angstrom Localization of Nanoparticles, Phys. Rev. Lett. 121, 193902 (2018).

[14] Lei Wei, Anatoly V. Zayats, and Francisco J. RodríguezFortuño, Interferometric Evanescent Wave Excitation of a Nanoantenna for Ultrasensitive Displacement and Phase Metrology, Phys. Rev. Lett. 121, 193901 (2018).

[15] Junaid Aasi, J. Abadie, B. P. Abbott, Richard Abbott, T. D. Abbott, M. R. Abernathy, Carl Adams, Thomas Adams, Paolo Addesso, and R. X. Adhikari, et al., Enhanced sensitivity of the ligo gravitational wave detector by using squeezed states of light, Nat. Photonics 7, 613 (2013).

[16] J. Abadie, Benjamin P. Abbott, R. Abbott, Thomas D. Abbott, M. Abernathy, Carl Adams, R. Adhikari, Christoph Affeldt, B. Allen, and G. S. Allen, et al., A gravitational wave observatory operating beyond the quantum shot-noise limit, Nat. Phys. 7, 962 (2011).

[17] Hans-Albert Bachor, Timothy C. Ralph, St Lucia, and Timothy C. Ralph, A Guide to Experiments in Quantum Optics (Wiley Online Library, Weinheim, 2004), Vol. 1.

[18] R. W. P. Drever, Gravitational Wave Detectors Using Laser Interferometers and Optical Cavities: Ideas, Principles and Prospects (Springer, Boston, MA, 1983).

[19] Brian J. Meers, Recycling in laser-interferometric gravitational-wave detectors, Phys. Rev. D 38, 2317 (1988).

[20] Kevin Lyons, Justin Dressel, Andrew N. Jordan, John C. Howell, and Paul G. Kwiat, Power-Recycled Weak-ValueBased Metrology, Phys. Rev. Lett. 114, 170801 (2015).

[21] Martin Neugebauer, Sergey Nechayev, Martin Vorndran, Gerd Leuchs, and Peter Banzer, Weak measurement enhanced spin hall effect of light for particle displacement sensing, Nano Lett. 19, 422 (2018).

[22] Lei Wei, Anatoly V. Zayats, and Francisco J. RodríguezFortuño, Interferometric Evanescent Wave Excitation of a Nanoantenna for Ultrasensitive Displacement and Phase 
Metrology, Phys. Rev. Lett. 121, 193901 (2018).

[23] Arseniy I. Kuznetsov, Andrey E. Miroshnichenko, Mark L. Brongersma, Yuri S. Kivshar, and Boris Luk'yanchuk, Optically resonant dielectric nanostructures, Science 354, 2472 (2016).

[24] Milton Kerker, D.-S. Wang, and C. L. Giles, Electromagnetic scattering by magnetic spheres, JOSA 73, 765 (1983).

[25] Vladislav Popov, Fabrice Boust, and Shah Nawaz Burokur, Constructing the near Field and far Field with Reactive Metagratings: Study on the Degrees of Freedom, Phys. Rev. Appl. 11, 024074 (2019).

[26] Younes Ra'di, Dimitrios L. Sounas, and Andrea Alù, Metagratings: Beyond the Limits of Graded Metasurfaces for Wave Front Control, Phys. Rev. Lett. 119, 067404 (2017).

[27] Zi-Lan Deng, Junhong Deng, Xin Zhuang, Shuai Wang, Tan Shi, Guo Ping Wang, Yao Wang, Jian Xu Yaoyu Cao, and Xiaolei Wang, et al., Facile metagrating holograms with broadband and extreme angle tolerance, Light: Sci. Appl. 7, 78 (2018).

[28] Ariel Epstein and Oshri Rabinovich, Unveiling the Properties of Metagratings via a Detailed Analytical Model for Synthesis and Analysis, Phys. Rev. Appl. 8, 054037 (2017).

[29] Jon A. Schuller, Rashid Zia, Thomas Taubner, and Mark L. Brongersma, Dielectric Metamaterials Based on Electric and Magnetic Resonances of Silicon Carbide Particles, Phys. Rev. Lett. 99, 107401 (2007).

[30] Wei Liu, Generalized Magnetic Mirrors, Phys. Rev. Lett. 119, 123902 (2017).
[31] Vladislav Popov, Fabrice Boust, and Shah Nawaz Burokur, Controlling Diffraction Patterns with Metagratings, Phys. Rev. Appl. 10, 011002 (2018).

[32] Alex M. H. Wong and George V. Eleftheriades, Perfect Anomalous Reflection with a Bipartite Huygens' Metasurface, Phys. Rev. X 8, 011036 (2018).

[33] V. S. Asadchy, A. Díaz-Rubio, S. N. Tcvetkova, D.-H. Kwon, A. Elsakka, M. Albooyeh, and S. A. Tretyakov, Flat Engineered Multichannel Reflectors, Phys. Rev. X 7, 031046 (2017).

[34] Steven M. Kay, Fundamentals of Statistical Signal Processing (Prentice Hall PTR, Upper Saddle River, New Jersey, 1993).

[35] Victor Twersky, On the scatttering of waves by an infinite grating, IRE Trans. Antennas Propagation 4, 330 (1956).

[36] Victor Twersky, On scattering of waves by the infinite grating of circular cylinders, IRE Trans. Antennas Propagation 10, 737 (1962).

[37] Bo Zhen, Chia Wei Hsu, Ling Lu, A. Douglas Stone, and Marin Soljačić, Topological Nature of Optical Bound States in the Continuum, Phys. Rev. Lett. 113, 257401 (2014).

[38] Ling Lu, John D. Joannopoulos, and Marin Soljacic, Topological photonics, Nat. Photonics 8, 821 (2014).

[39] Chia Wei Hsu, Bo Zhen, Jeongwon Lee, Song-Liang Chua, Steven G. Johnson, John D. Joannopoulos, and Marin Soljacic, Observation of trapped light within the radiation continuum, Nature 499, 188 (2013).

[40] M. Z. Hasan and C. L. Kane, Colloquium: Topological insulators, Rev. Mod. Phys. 82, 3045 (2010). 